S. Kwok, M. Dopita, and R. Sutherland, eds.

\title{
The Molecular Morphology of Evolved Stars
}

\author{
D. Fong, M. Meixner, E.C. Sutton \\ University of Illinois, 1002 W. Green St., Urbana, Il. 61801, USA
}

W.J. Welch

University of California, Radio Astronomy Lab, Berkeley, Ca. 94720

\begin{abstract}
We present ${ }^{12} \mathrm{CO} \mathrm{J}=1-0$ observations of a small sample of evolved stars to investigate the evolution of the molecular envelope from the AGB phase to the formation of the PN.
\end{abstract}

The history of the circumstellar gas is imprinted on the circumstellar envelope itself, such as the record of its molecular mass loss, and its interaction with fast winds and dissociating/ionizing photons. By imaging the morphology and the kinematic structure, we can model the mass loss history and determine how fast winds, shocks and photodissociation/photoionization have transformed these envelopes. The morphology around most AGB stars are roughly spherical, but the shapes of most PNe deviate from this symmetry. Exactly when and how this asymmetry forms is still not known. Our study offers more insight into the beginnings of this morphological transition.

By combining data from the Berkeley-Illinois-Maryland-Association (BIMA) Millimeter Array and the NRAO 12m, we have constructed full synthesis data cubes for MIRA, IRC +10216, HD 161796 (IRAS 17436+5003), AFGL 2688 (Egg Nebula), IRAS 22272+5435 (HD 235858), AFGL 2343 (IRAS 19114+0002) and NGC 7027. Figure 1 shows the variety of morphologies that we are encountering in the sample. All the envelopes exhibit various degrees of deviation from spherical symmetry. The PN, NGC 7027, contains a photodissociated hole and a bright ring with breaks along the symmetry axis. The PPN, AFGL 2688, contains extended emission that is elliptically shaped, but evidence for collimated structures the are seen in the inner regions. The global morphology of the AGB star, IRC+10216, is essentially spherical which suggest that the early mass loss was spherical in nature, but more recently the geometry of the mass loss has changed because an elongated N-S feature appears to be emerging from the central regions. This suggest that the onset of the asymmetry has begun before the star has left the AGB phase. 

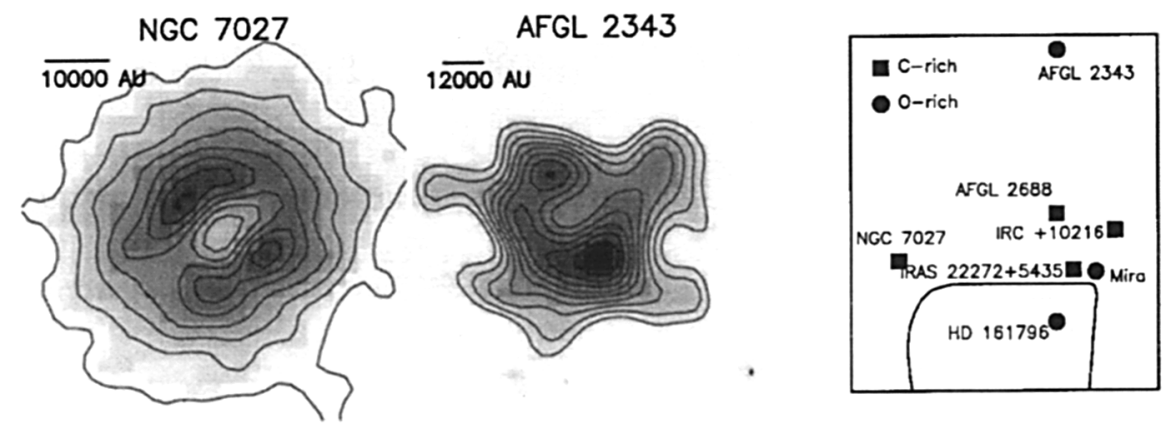

AFGL 2688

)

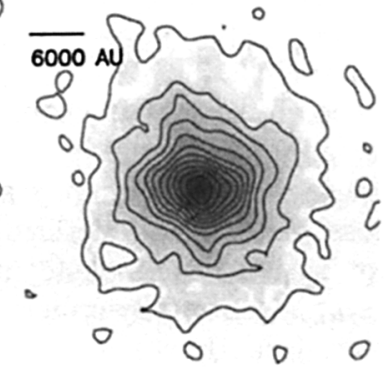

IRAS $22272+5435$
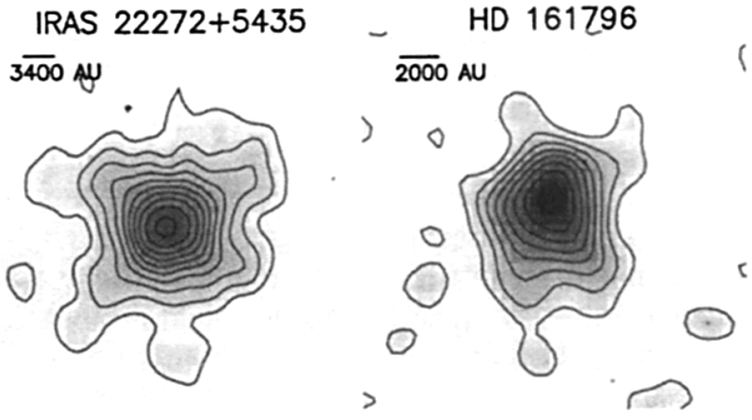

$$
\mathrm{IRC}+10216
$$

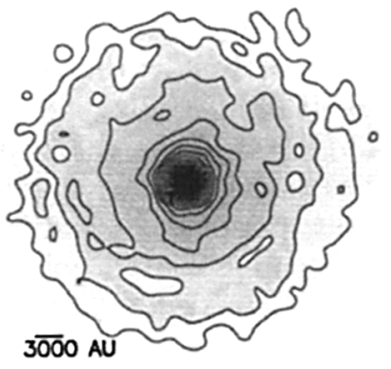

MIRA

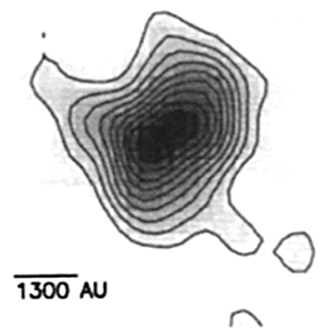

Figure 1. The ${ }^{12} \mathrm{CO} \mathrm{J}=1-0$ maps display the systemic velocity of the sources with $1-2 \mathrm{~km} \mathrm{~s}^{-1}$ widths. The HR diagram at the top right panel shows the evolutionary distribution of the sample. 